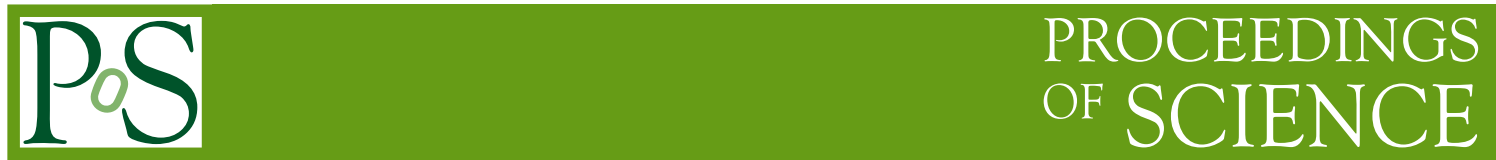

\title{
PDFs for Higgs Physics
}

\section{Joey Huston*}

Michigan State University, East Lansing, MI USA 48824

huston@msu.edu

PDF $+\alpha_{s}\left(m_{Z}\right)$ benchmarking exercises for Higgs production at the LHC are described, along with attempts to improve on the resultant uncertainties.

11th International Symposium on Radiative Corrections (Applications of Quantum Field Theory to Phenomenology) (RADCOR 2013),

22-27 September 2013

Lumley Castle Hotel, Durham, UK

*I would like to thank my colleagues from the benchmarking papers whose results are summarized in this proceeding. 


\section{Introduction}

To fully understand the properties of the Higgs boson observed at the LHC, it is crucial to have good control over the theoretical uncertainties on the predictions for its production cross sections in the various sub-processes used in its measurement. One of the most important theoretical uncertainties is that due to an incomplete knowledge of the parton distribution functions (PDFs) that contribute to the Higgs boson production cross sections, along with the accompanying uncertainties on the value of the strong coupling constant $\alpha_{s}\left(m_{Z}\right)$ to be used in the calculation of the cross sections.

In 2010, an exercise was carried out to which all PDF groups were invited to participate [1]. The exercise consisted of comparing next-to-leading (NLO) predictions, using PDFs from each group, for several benchmark Standard Model cross sections at a center-of-mass energy of $7 \mathrm{TeV}$. Each PDF group determines PDF uncertainties within its own framework (such as Hessian or Monte Carlo). The uncertainties derive primarily from the uncertainties of the data sets used in the fitting of the PDFs. The central PDF, and the PDF uncertainties, derived by each group can differ due to issues such as the selection of data used in the PDF fits, parameterization biases, and differences in theoretical treatments, etc.

Based on the benchmarking exercise, PDF4LHC recommendations were developed, at NLO and NNLO, to provide a better estimate of the real PDF uncertainty, taking into account differences in theoretical treatments in addition to the uncertainties of the input experimental data sets [2]. The results of these benchmarking exercises were also included in the Higgs Yellow Reports [3, 4, 5].

A followup benchmarking exercise was carried out (primarily at NNLO) in 2013, using updated versions of the PDFs available in the earlier exercise [6]. Some of the results of this exercise are discussed below.

\section{PDF luminosities}

Perhaps the easiest way to present the PDF sensitivity for any given initial state is to plot the parton luminosity function, which following Ref. [7] is defined as

$$
\Phi_{i j}\left(M_{X}^{2}\right)=\frac{1}{s} \int_{\tau}^{1} \frac{d x_{1}}{x_{1}} f_{i}\left(x_{1}, M_{X}^{2}\right) f_{j}\left(\tau / x_{1}, M_{X}^{2}\right),
$$

where $f_{i}\left(x, M^{2}\right)$ is a PDF at a scale $M^{2}$, and $\tau \equiv M_{X}^{2} / s$.

In the benchmarking paper (and in this contribution), all of the PDFs are compared at a common value of $\alpha_{s}\left(m_{Z}\right)$ of 0.118 , and the parton luminosities are displayed as a ratio to that of the NNPDF2.3 parton luminosity, for a center-of-mass energy of $8 \mathrm{TeV}$. The PDF uncertainties are evaluated at the $68 \%$ confidence level. To study the effect of varying the value of $\alpha_{s}\left(m_{Z}\right)$, results are also provided for two values of $\alpha_{s}\left(M_{Z}\right), \alpha_{s}=0.117$ and 0.119 . The motivation for this choice is that these values approximately bracket the current 2012 PDG best fit value [8], $\alpha_{s}\left(M_{Z}\right)=0.1184 \pm 0.0007$. They also include the preferred or best-fit $\alpha_{s}$ values of CT10, MSTW08 and NNPDF2.3 at NNLO.

The quark-quark and quark-antiquark luminosities and their uncertainties are shown in Fig. 1 and the gluon-gluon and gluon-quark luminosities and their uncertainties are shown in Fig. 2. There 
is a good agreement between the CT10 [9] ${ }^{1}$, MSTW08 [10] and NNPDF2.3 [11] quark-quark and quark-antiquark PDF luminosities; in fact there is almost a $100 \%$ overlap in the mass range appropriate for $W / Z$ production. The ABM11 [12] luminosities tend to be higher at low mass, and to fall off more rapidity at high mass. The HERAPDF1.5 $[13,14]$ luminosities are consistent with those from CT10, MSTW08 and NNPDF2.3, but with larger uncertainties due to the reduced data set used in the fit.
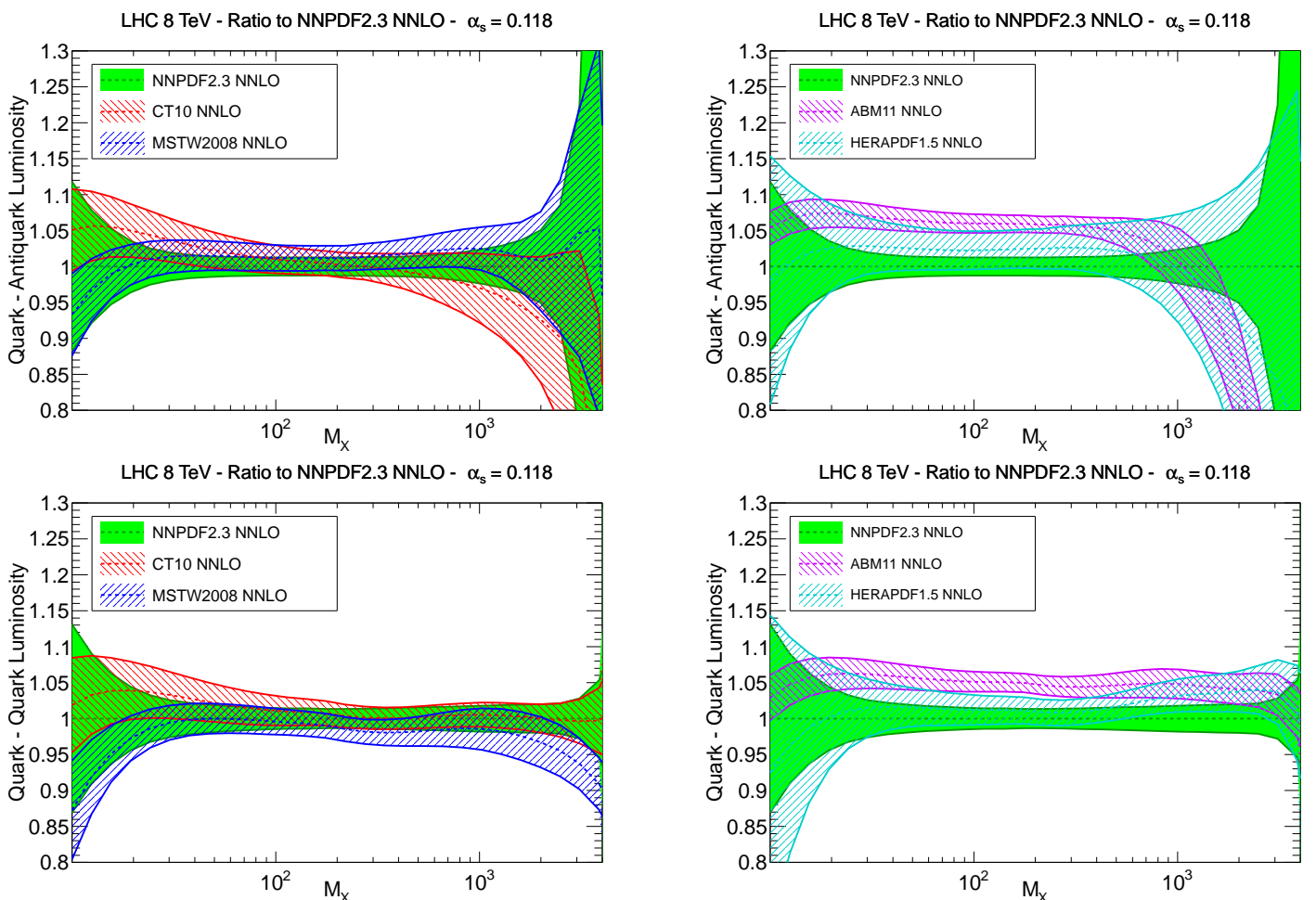

Figure 1: The quark-antiquark (upper plots) and quark-quark (lower plots) luminosities, Eq. (2.1), for the production of a final state of invariant mass $M_{X}$ (in $\mathrm{GeV}$ ) at LHC $8 \mathrm{TeV}$. The left plots show the comparison between NNPDF2.3, CT10 and MSTW08, while the right plots compare NNPDF2.3, HERAPDF1.5 and MSTW08. All luminosities are computed at a common value of $\alpha_{s}=0.118$.

The gluon-gluon luminosities are in reasonable, but not perfect, agreement between CT10, MSTW08 and NNPDF2.3 (and HERAPDF within their larger uncertainties). The gluon-gluon luminosity for ABM11 is significantly smaller than for the other PDFs at high mass. The size of the gluon-gluon luminosity uncertainties are almost exactly the same, in the region appropriate for Higgs boson production, among CT10, MSTW08 and NNPDF2.3 (see Fig. 3), but the central predictions are not, leading to a larger range of luminosity uncertainty (basic ally a factor of 2) in the region for Higgs production, if the full limits of the three uncertainty bands are used. Given the importance of the $g g$ fusion sub-process, it is clearly desirable to reduce this uncertainty if possible.

\footnotetext{
${ }^{1}$ The CT10 PDF uncertainties are normally provided at the $90 \%$ confidence level, and are scaled to a $68 \%$ confidence level by dividing by a factor of 1.645 .
} 
LHC $8 \mathrm{TeV}$ - Ratio to NNPDF2.3 NNLO $-\alpha_{\mathrm{s}}=0.118$

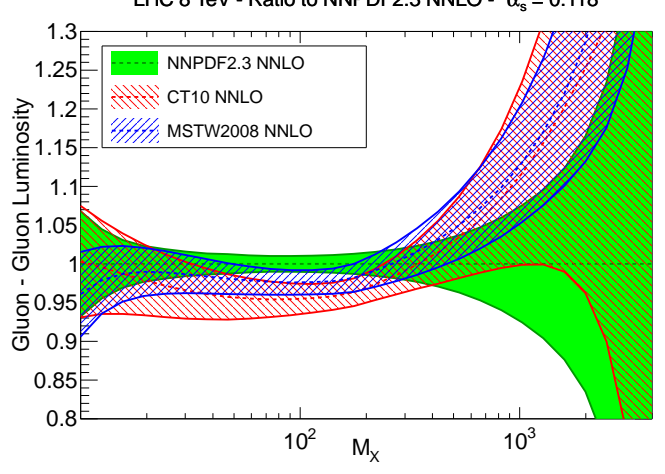

LHC 8 TeV - Ratio to NNPDF2.3 NNLO $-\alpha_{s}=0.118$

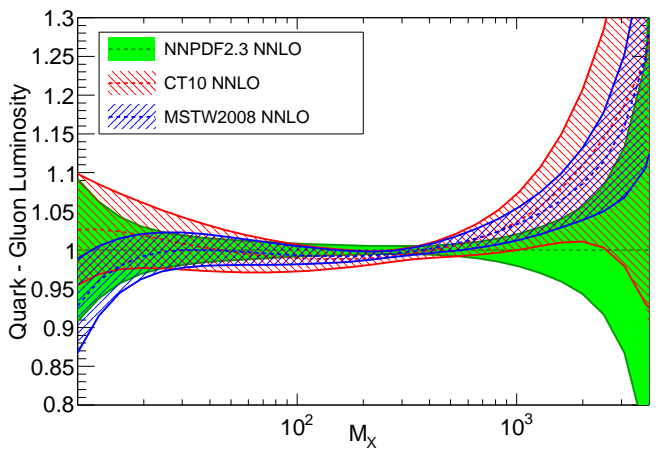

LHC $8 \mathrm{TeV}$ - Ratio to NNPDF2.3 NNLO - $\alpha_{s}=0.118$

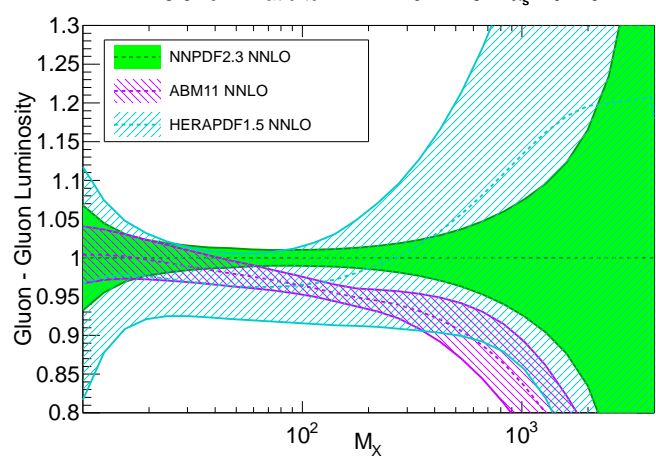

LHC $8 \mathrm{TeV}$ - Ratio to NNPDF2.3 NNLO $-\alpha_{\mathrm{s}}=0.118$

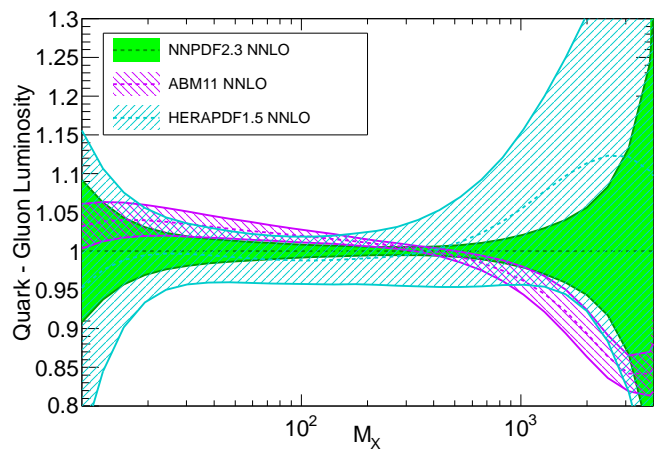

Figure 2: Same as Fig. 1 for the gluon-gluon (upper plots) and quark-gluon (lower plots) luminosities.

\section{Higgs cross sections}

Next, the NNLO cross sections for Higgs production through $g g$ fusion are calculated at 8 TeV using CT10, MSTW08 and NNPDF2.3. The cross sections are computed at NNLO with the iHixs code [15]. The central scale has been taken to be $Q=m_{H}$, following the recommendations of the Higgs Cross Section Working Group (HXSWG), and the cross sections have been calculated at two values of $\alpha_{s}\left(m_{Z}\right), 0.117$ and 0.119 . The hierarchy of calculated Higgs boson cross sections (at each value of $\alpha_{s}\left(m_{Z}\right)$ shown in Fig. 4 reproduces that found with the PDF luminosities alone. For reference, the cross sections for $g g$ fusion are shown, along with those from other Higgs boson production processes, in Table 1.

As an exercise, the PDF $+\alpha_{s}\left(m_{Z}\right)$ uncertainty is calculated using the three PDFs, combining the PDF and $\alpha_{s}\left(m_{Z}\right)$ uncertainties in quadrature. The uncertainty on $\alpha_{s}$ is taken to be $\delta \alpha_{s}=0.0012$ at the $68 \%$ confidence level. The central value is taken as the midpoint of the envelope defined in this way.(This differs somewhat from the 2010 PDF4LHC prescription in that in the latter, the prediction for each of the PDF sets uses the native value of $\alpha_{s}\left(m_{Z}\right)$ for that set.)

Using the NNLO cross sections from these 3 NNLO PDF sets, the cross section with its uncertainty is given by

$$
\sigma_{H}^{N N L O}=18.75 \pm 1.24 \mathrm{pb}, \quad\left(6.6 \% " \mathrm{PDF}+\alpha_{s}{ }^{\prime}\right) .
$$

The central value is within 2\% from the MSTW2008 NNLO value of $18.45 \mathrm{pb}$, which in the 2010 

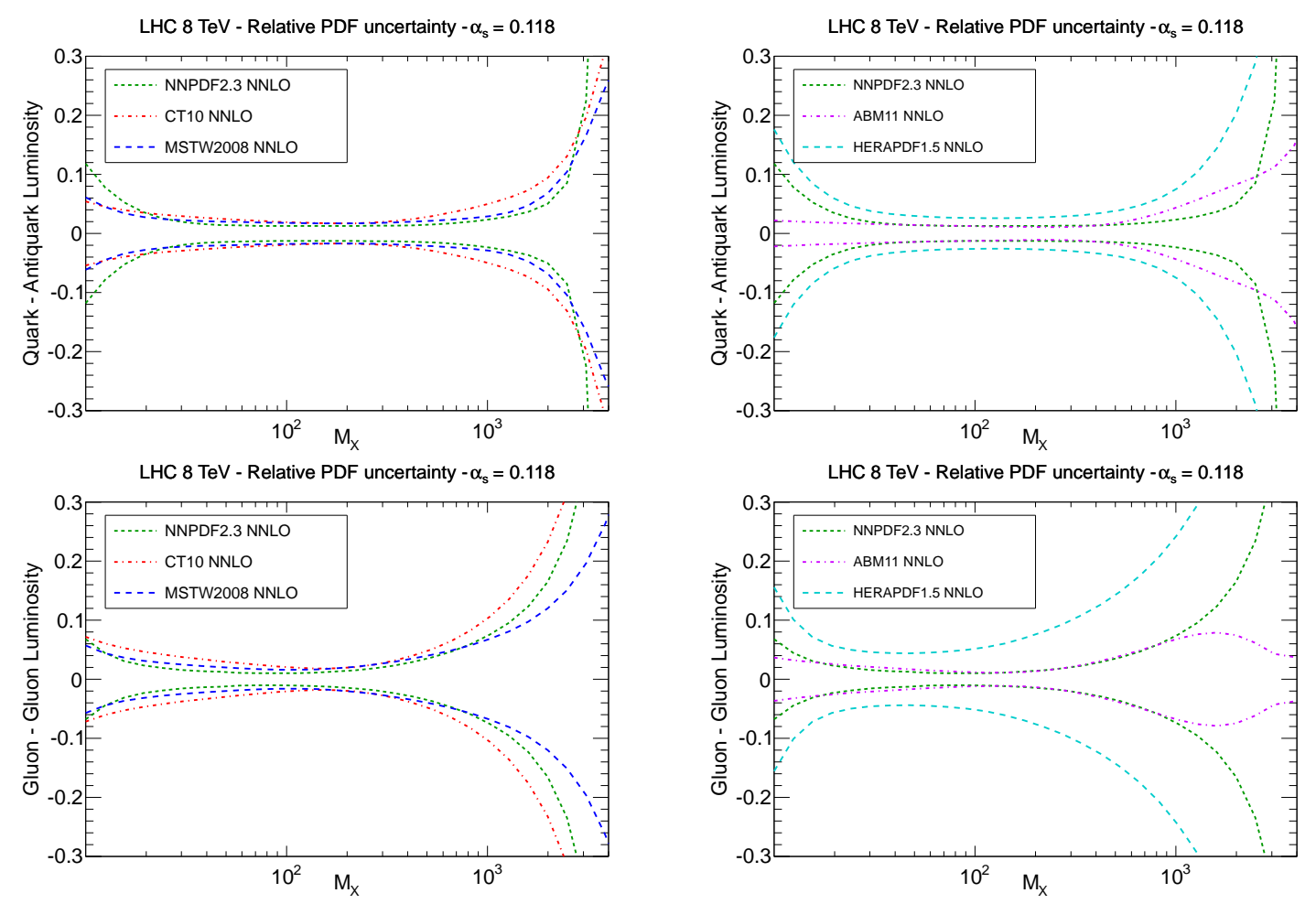

Figure 3: The relative PDF uncertainties in the quark-antiquark luminosity (upper plots) and in the gluongluon luminosity (lower plots), for the production of a final state of invariant mass $M_{X}$ (in GeV) at the LHC $8 \mathrm{TeV}$. All luminosities are computed at a common value of $\alpha_{s}=0.118$.

PDF4LHC NNLO prescription was taken as the central value. A similar combination carried out at NLO results in a similar uncertainty as that found at NNLO.

The NNLO cross section for Higgs production at LHC $(8 \mathrm{TeV})$ is currently quoted by the HXSWG $^{2}$ as

$$
\sigma_{H}^{\mathrm{NNLO}}=19.52 \pm 1.41 \mathrm{pb}, \quad\left( \pm 7.2 \% " \mathrm{PDF}+\alpha_{s}{ }^{\prime}\right) .
$$

The HXSWG cross section numbers have been computed with the current (2010) PDF4LHC prescription, $m_{H}=125 \mathrm{GeV}$, and the de Florian-Grazzini code [16], which incorporates soft-gluon effects up to next-to-next-to-leading logarithmic accuracy on top of the exact NNLO calculation. The resulting cross sections are larger than those obtained from the purely fixed-order results used in this benchmarking study. The percentage uncertainties for the combined PDF $+\alpha_{s}\left(m_{Z}\right)$ variations, though, can be directly compared with those determined from the benchmark exercise. The two agree well, with the new benchmark uncertainties being slightly smaller.

\section{Conclusions}

In this contribution, I have briefly summarized the most recent PDF benchmarking exercise for Higgs boson production at the LHC, concentrating on the $g g$ fusion channel. The best estimate for

\footnotetext{
${ }^{2}$ https://twiki.cern.ch/twiki/bin/view/LHCPhysics/CERNYellowReportPageAt8TeV
} 


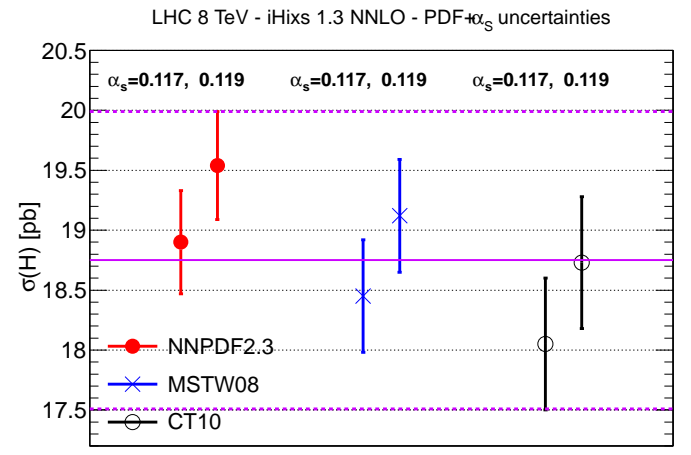

Figure 4: The cross sections for Higgs boson production in the $g g$ fusion channel are plotted using the CT10, MSTW08 and NNPDF2.3 PDF sets. The cross sections are plotted for two values of $\alpha_{s}\left(m_{Z}\right), 0.117$ and 0.119. The Higgs boson production cross section in the gluon fusion channel using the NLO PDF sets included in the PDF4LHC prescription for $\alpha_{s}=0.117$ and 0.119 . The envelope (dashed violet horizontal lines) is defined by the upper and lower values of the predictions from all the three PDF sets and the two values of $\alpha_{s}$. The solid violet horizontal line is the midpoint of the envelope.

\begin{tabular}{c||c|c|c|c|c}
\hline \multicolumn{6}{c}{ Gluon Fusion $(\mathrm{pb})$} \\
\hline$\alpha_{S}\left(M_{Z}\right)$ & NNPDF2.3 & MSTW08 & CT10 & ABM11 & HERAPDF1.5 \\
\hline \hline 0.117 & $18.90 \pm 0.20$ & $18.45 \pm 0.24$ & $18.05 \pm 0.36$ & $18.11 \pm 0.41$ & $18.34 \pm 1.03$ \\
0.119 & $19.54 \pm 0.25$ & $19.12 \pm 0.25$ & $18.73 \pm 0.37$ & $18.71 \pm 0.42$ & $18.94 \pm 1.07$ \\
\hline
\end{tabular}

\begin{tabular}{c||c|c|c|c|c}
\hline \multicolumn{7}{c}{ Vector Boson Fusion $(\mathrm{pb})$} \\
\hline$\alpha_{S}\left(M_{Z}\right)$ & NNPDF2.3 & MSTW08 & CT10 & ABM11 & HERAPDF1.5 \\
\hline \hline 0.117 & $1.635 \pm 0.020$ & $1.655 \pm 0.029$ & $1.681 \pm 0.030$ & $1.728 \pm 0.020$ & $1.668 \pm 0.051$ \\
0.119 & $1.644 \pm 0.020$ & $1.658 \pm 0.029$ & $1.686 \pm 0.030$ & $1.731 \pm 0.020$ & $1.673 \pm 0.051$ \\
\hline
\end{tabular}

\begin{tabular}{c||c|c|c|c|c}
\hline \multicolumn{6}{c}{$W H$ production $(\mathrm{pb})$} \\
\hline$\alpha_{S}\left(M_{Z}\right)$ & NNPDF2.3 & MSTW08 & CT10 & ABM11 & HERAPDF1.5 \\
\hline \hline 0.117 & $0.739 \pm 0.010$ & $0.746 \pm 0.011$ & $0.738 \pm 0.016$ & $0.784 \pm 0.010$ & $0.751 \pm 0.023$ \\
0.119 & $0.747 \pm 0.010$ & $0.752 \pm 0.011$ & $0.745 \pm 0.016$ & $0.789 \pm 0.010$ & $0.754 \pm 0.023$ \\
\hline
\end{tabular}

\begin{tabular}{c||c|c|c|c|c}
\hline \multicolumn{6}{c}{$t \bar{t} H$ associated production $(\mathrm{fb})$} \\
\hline$\alpha_{S}\left(M_{Z}\right)$ & NNPDF2.3 & MSTW08 & CT10 & ABM11 & HERAPDF1.5 \\
\hline \hline 0.117 & $72.8 \pm 2.1$ & $74.6 \pm 1.6$ & $71.6 \pm 3.4$ & $66.6 \pm 2.0$ & $76.2 \pm 9.0$ \\
0.119 & $75.1 \pm 2.0$ & $77.3 \pm 1.6$ & $76.1 \pm 3.4$ & $69.4 \pm 2.0$ & $79.4 \pm 9.0$ \\
\hline
\end{tabular}

Table 1: The Higgs boson production cross sections (in pb) in various Higgs boson production channels (from top to bottom: gluon fusion, vector boson fusion, $W H$ production and $t \bar{t} H$ production), for $m_{H}=$ $125 \mathrm{GeV}$ at LHC $8 \mathrm{TeV}$. Results for the CT10, MSTW08 and NNPDF2.3 PDFs are shown, along with those for ABM11 and HERAPDF1.5.

the PDF uncertainty in the $g g$ channel is still roughly a factor of two larger than the uncertainties determined by any of the 3 PDFs used to construct that global uncertainty, in contrast for example to the $W / Z$ cross section predictions. Given the importance of this channel towards a quantitative understanding of Higgs boson production, it is important to understand whether that uncertainty 
can be improved. The gluon distribution in the relevant range is determined primarily by the deepinelastic (DIS) data from HERA and from several fixed target DIS experiments. New data sets from the LHC sensitive to the gluon distribution may help to improve the situation, but given the relatively small discrepancy that we are attempting to resolve, there are great demands on the needed systematic and statistical uncertainties for the LHC data. It may also be possible to reduce the spread of the gluon-gluon luminosity uncertainty by a detailed examination of the theoretical frameworks for each PDF fit. Such an exercise is currently underway in the context of the Les Houches workshop (http://phystev.in2p3.fr/Houches2013/).

Even if the $g g$ PDF luminosity uncertainty can be decreased, there is still a substantial $\alpha_{s}\left(m_{Z}\right)$ uncertainty. A discussion of possible improvements in the knowledge of $\alpha_{s}\left(m_{Z}\right)$ can be found in the Snowmass QCD report [17].

Complete NNLO and NLO benchmark results from the study discussed in this contribution can be obtained online from HepForge:

\section{http://nnpdf.hepforge.org/html/pdfbench/catalog.}

I would like to thank the organizers for the excellent physics program and for what may be the most interesting workshop venue that I have ever been to. I would also like to publicly forgive Lance Dixon for not protecting my flank during the paintball excursion.

\section{References}

[1] S. Alekhin, S. Alioli, R. D. Ball, V. Bertone, J. Blumlein, M. Botje, J. Butterworth and F. Cerutti et al., arXiv:1101.0536 [hep-ph].

[2] M. Botje, J. Butterworth, A. Cooper-Sarkar, A. de Roeck, J. Feltesse, S. Forte, A. Glazov and J. Huston et al., arXiv:1101.0538 [hep-ph].

[3] S. Dittmaier et al. [LHC Higgs Cross Section Working Group Collaboration], arXiv:1101.0593 [hep-ph].

[4] S. Dittmaier, S. Dittmaier, C. Mariotti, G. Passarino, R. Tanaka, S. Alekhin, J. Alwall and E. A. Bagnaschi et al., arXiv:1201.3084 [hep-ph].

[5] SHeinemeyer et al. [LHC Higgs Cross Section Working Group Collaboration], arXiv:1307.1347 [hep-ph].

[6] R. D. Ball, S. Carrazza, L. Del Debbio, S. Forte, J. Gao, N. Hartland, J. Huston and P. Nadolsky et al., JHEP 1304 (2013) 125 [arXiv:1211.5142 [hep-ph]].

[7] J. M. Campbell, J. W. Huston and W. J. Stirling, Rept. Prog. Phys. 70 (2007) 89 [hep-ph/0611148].

[8] Particle Data Group, J. Beringer et al., Phys.Rev. D86 (2012) 010001.

[9] P. Nadolsky et al., (2012), arxiv:1206.3321.

[10] A.D. Martin et al., Eur. Phys. J. C63 (2009) 189, arxiv:0901.0002.

[11] R.D. Ball et al., Nucl.Phys. B867 (2013) 244, arxiv:1207.1303.

[12] S. Alekhin, J. Blumlein and S. Moch, (2012), arxiv:1202.2281.

[13] H1 and ZEUS Collaborations, V. Radescu, PoS ICHEP2010 (2010) 168. 
[14] ZEUS Collaboration, H1 Collaboration, A. Cooper-Sarkar, PoS EPS-HEP2011 (2011) 320, arxiv:1112.2107.

[15] C. Anastasiou et al., JHEP 1112 (2011) 058, 1107.0683.

[16] D. de Florian, M. Grazzini and M. Grazzini, Phys.Lett. B718 (2012) 117, arxiv:1206.4133.

[17] J. M. Campbell, K. Hatakeyama, J. Huston, F. Petriello, J. R. Andersen, L. Barze, H. Beauchemin and T. Becher et al., arXiv:1310.5189 [hep-ph]. 